Review article

Central Eur J Paed 2017;13(1):12-23

DOI $10.5457 / \mathrm{p} 2005-114.165$

\title{
VACCINATION AND AUTOIMMUNE PHENOMENA
}

\author{
Srdja JANKOVIĆ \\ University Children's Hospital \\ Belgrade, Serbia
}

\author{
Correspondence: \\ ysrdja@gmail.com \\ Tel.: + 381112060674 \\ Fax.: + 381113621413
}

Received: December 15, 2017

Accepted: February 17, 2017

Key words: Vaccines • Vaccine safety • Autoimmunity - Autoimmune disorders.
The purpose of this review is to provide an up-to-date outlook on autoimmune phenomena associated with vaccination. Autoimmune reactions triggered by vaccination are a rare occurrence, but a frequent cause of concern. Search of available medical literature for terms "vaccination" and "autoimmunity" or "autoimmune" was performed. Additional articles were indentified based on citations in retrieved articles. Initiation of an autoimmune response by vaccination is possible, but rare. Potential mechanisms include molecular mimicry and bystander activation. In addition, adjuvants present in vaccines may trigger an autoimmune response in specifically predisposed persons by stimulating innate immunity. Documented instances of vaccine-related autoimmunity include Guillain-Barré syndrome following 1976 pandemic influenza (New Jersey strain) vaccine, immune thrombocytopenic purpura triggered by measles, mumps and rubella (MMR) vaccine, and myopericarditis associated with smallpox vaccine. Many autoimmune phenomena have been reported to have occurred in individual patients, or series of patients, in temporal association with the administration of a vaccine. However, controlled studies in most such instances either failed to confirm a causal relationship or were not performed. Conclusion - The weight of currently available evidence supports the conclusion that any risks of autoimmune reactions to vaccines are far outweighed by vaccine-afforded protection against infections, particularly in view of the fact that infections, including vaccine-preventable infections, are most important triggers of autoimmunity. However, research into autoimmune reactions apparently initiated by vaccines is potentially of great importance in the continuing quest to improve scientific understanding of how the immune system functions in health and disease, as well as to widen the evidence base for future efforts directed toward additional improvement in vaccine safety.

\section{Introduction}

Active immunization (vaccination) is, beyond doubt, an extremely important method of prevention of infectious diseases and has repeatedly been proven to be quite safe as well as highly effective. This, however, does not signify that vaccination is absolutely devoid of any potential health risks. Among these, the possibility of initiating or exacerbating an autoimmune process by active immunization has been the subject of much attention and continuing intense scrutiny. There are many individual reports and case series of temporal association between vaccination and initiation or exacerbation of an autoimmune disease in the literature. A monograph on proposed associations of vaccines with auto- 
immunity has been published by Shoenfeld, Agmon-Levin and Tomljenovic (1). However, no more than a tiny fraction of these putative associations are confirmed by subsequent methodologically rigorous research. The weight of scientific evidence supports the conclusion that vaccines are, by orders of magnitude, less frequent triggers of autoimmune processes than are infectious diseases, including vaccine-preventable diseases. This is in accordance with many reports of slightly decreased incidence of autoimmune disorders (AID) in vaccinated persons, compared to non-vaccinated controls (2). Another matter of contention is the question of risk of triggering an exacerbation of existing autoimmune disease by vaccination. This has, so far, been shown to be far too low to warrant withholding vaccination from such patients in the absence of specific contraindications, all the more so because, again, infections, including vaccine-preventable infections, can easily be demonstrated as one of key factors behind disease exacerbations (3). In this paper, we will review the current evidence on autoimmune phenomena induced by vaccination and their relevance for the overall riskbenefit analysis of vaccination.

\section{Vaccination-Induced Autoimmunity: Potential Mechanisms}

In essence, the potential of vaccines to trigger autoimmunity is largely the consequence of the fact that vaccines, in order to be effective, must elicit some (although preferably subclinical) degree of inflammatory reaction, i.e., some degree of non-specific stimulation of the immune system, often achieved by the addition of adjuvants (4). There are two main groups of potential mechanisms of initiating an autoimmune response by vaccination at the level of adaptive immunity: molecular mimicry and bystander activation. The latter group includes non-specific effects of vac- cines upon innate immunity that could plausibly lead to an overt autoimmune reaction in an appropriately predisposed individual and/ or create an environment favorable to amplification and/or perpetuation of an existing silent or low-level autoimmune process.

The possibility to inadvertently induce, trigger or exacerbate autoimmune responses by adjuvants used in vaccines has been the focus of extensive research conducted on animal models. Some models designed for this purpose have recently been described by Ruiz et al. (5). There are many reports of successful induction or exacerbation of autoimmunity by adjuvants. For instance, mice have been reported to have developed autoantibodies after intraperitoenal injection of hydrocarbon oil-based adjuvants (6). In another murine model, concomitant exposure to HPV vaccine and pertussis toxin has reportedly resulted in hypothalamic destruction (7). Similarly, exposure to HBV vaccine has been found to accelerate disease progression in a murine model of lupus (8). A number of published papers has also expressed concern regarding the biopersistence and possible neutotoxicity of aluminium-based adjuvants, with some apparent support from animal models $(9,10,11)$. The question of relevance of these examples to adjuvant/vaccine safety assessment is, however, not to be taken lightly. The effects of adjuvants on the immune system are well known to depend, in addition to the type of adjuvant, on the applied dose, route of administration, duration and/or frequency of exposure, and, of course, the recipient species, not allowing direct extrapolation from animal models to humans. In view of the fact that instances of adjuvantassociated autoimmunity have been shown by an impressive amount of experience to be quite rare, and that extensive research has so far confirmed a fairly good safety profile of all adjuvants that are currently in use in human vaccines $(12,13,14)$, animal model data on 
potential adjuvant-associated autoimmunity need to be interpreted with extreme caution and may only be used to inform further research.

Molecular mimicry is a known pathogenic mechanism behind some complications of infectious disorders, such as rheumatic fever and neuroborreliosis. Since all vaccines must necessarily contain one or more antigens specific to the microorganism that is to be prevented from causing infection, the theoretical possibility of molecular mimicry between antigens belonging to the host and vaccine components cannot, in principle, be excluded. Clearly established examples are, however, rare rather than common. Well known instances include gangliosides that are targeted in Guillain-Barré syndrome (GBS), with structural homologues present in a number of bacterial and viral vaccines (15), as well as the homology between hepatitis B virus polymerase peptide and myelin components, as shown in the model of autoimmune encephalomyelitis in rabbits (16). Many more examples of epitopes of vaccinal antigens displaying a certain degree of homology to parts of human proteins have been reported, as extensively reviewed in a recent article by Kanduc and Shoenfeld (17) for HBV and $\mathrm{HPV}$ vaccines. There are also many more examples of potential mimicry epitopes identified in animal studies. Documented existence of molecular mimicry between self and vaccine components does not, however, by itself indicate that an autoimmune reaction will be elicited by the vaccine. For example, the structural homology between Borrelia burgdorferi protein $\mathrm{A}$ and human lymphocyte function-associated antigen (LFA)- 1 is not accompanied by any reports of arthritis after the administration of vaccine against Lyme disease containing the protein in question (18), even though this particular instance of molecular mimicry is highly likely to be involved in the pathogenesis of arthritis aris- ing as part of the natural course of borreliosis (19). In a similar way, an autoimmune response induced by tetanus toxoid in mice has been shown to be qualitatively different from the response to the same autoantigen found in autoimmune disease (20).

Two examples of molecular mimicry between human tissues and vaccine components commanded particular attention due to obvious potential clinical relevance: structural similarity between the small hepatitis B surface antigen and human myelin components (21) and that between HPV 16 major capsid L1 protein and certain human proteins involved in cardiac function (22). However, the incidence of related autoimmune disorders (demyelinating diseases and cardiac rhytm abnormalities, respectively) was not found to be detectably increased by the administration of these vaccines (see below).

Bystander activation denotes activation of $\mathrm{T}$ cell clones not carrying antigen receptors specific to antigen(s) that elicited the immune response. To a certain extent, this occurs as part of any reaction of the immune system to a specific stimulus. The concern that this could lead to activation of some autoreactive $T$ cell clones in the settings of vaccination is therefore theoretically valid. However, activation of such autoreactive clones is, in itself, not sufficient to cause an overt autoimmune reaction, or trigger the onset of an AID. This would require a deeper dysregulation of the immune system, with loss or dysfunction of multiple physiological protective mechanisms. Furthermore, ample experimental evidence suggests that bystander activation of $\mathrm{T}$ cells usually requires tissue damage in the target organ, i.e., pro-inflammatory environment. This can be explained by the abundance of damage-associated molecular patterns (DAMP) in the affected tissue. These DAMP bind to receptors on innate immune cells, and these cells in turn increase activatory signaling directed at $\mathrm{T}$ lymphocytes, poten- 
tially reducing the threshold for an autoreactive response. Vaccines are designed to keep the release of DAMP to the minimum, while infectious diseases are often accompanied by tissue damage resulting in an abundance of DAMP. This is plausibly one of the reasons why autoimmune reactions triggered by infections have a strong tendency to be more frequent, as well as more severe, than autoimmune reactions triggered by vaccines (23).

The appearance of autoantibodies, presumably a result of bystander activation, in humans after immunization has been observed and is, in itself, not a rare phenomenon. For instance, one study in Slovenia found a (most often transient) autoantibody response after influenza vaccination in approximately $15 \%$ of 92 healthy adults studied (24). Again, in the absence of a (rare) individual predisposition, or a deeper dysregulation of the immune system with insufficiency or failure of key physiological protective mechanisms, the presence of autoantibodies or autoreactive cells can by no means initiate an autoimmune reaction.

\section{Vaccination-Induced Autoimmunity: Confirmed Causal Connection}

The following are some of the examples featuring reasonable evidence of a causal connection between a vaccine and an autoimmune manifestation. The list is not exhaustive.

\section{Encephalomyelitis and Rabies Vaccine}

A historically important, though no longer practically relevant example of vaccine-induced autoimmune reaction was related to an early form of rabies vaccine that caused disseminated encephalomyelitis in $-0.1 \%$ of vaccinees (25). While this almost certainly represented an immunological adverse reaction with serious consequences, this risk is not shared to anywhere nearly the same degree by modern vaccines.

\section{Guillain-Barré Syndrome and Influenza Vaccination}

Guillain-Barré syndrome (GBS) comprises a group of acute demyelinating polyradiculoneuropathies with several distinct clinical forms. The majority of cases of GBS are triggered by infection, most notably Campylobacter jejuni (26). Several viruses, including influenza virus, are also known to trigger GBS (27). It is thought that autoantibodies directed at gangliosides, elicited by molecular mimicry involving components of microorganisms, play a role in the pathogenesis of GBS. Occurrence of GBS has been sporadically reported in association with several vaccines (28). Causal connection has, however, been undisputably established solely for the 1976 swine influenza (A/New Jersey/76) vaccine. During the influenza pandemic that struck the world in that year, 40 million persons were vaccinated in the United States alone, with GBS reported as a complication in approximately 1:100,000, an estimated five- to ten-fold increase against baseline incidence (29). The connection of GBS with other influenza vaccines is far less clear (30).

\section{Demyelinating CNS disorders}

A wide variety of demyelinating CNS disorders have been reported to be temporally associated with one or more vaccines, most prominently acute disseminated encephalomyelitis, optic neuritis, or myelitis. A recent review found 71 cases of a demyelinating disorder occurring after administration of a vaccine for the period 1979-2013 (31). This translates to an estimated incidence of about one in a million vaccinations. Another review, looking specifically at vaccination against $\mathrm{HBV}$, previously reported in several case series to be associated with CNS autoimmunity, reached a similar conclusion (32). 


\section{Immune Thromobocytopenic Purpura and MMR vaccination}

Immune thrombocytopenic purpura (ITP) is known to be associated with MMR vaccine in children, with reported incidence of $1-3$ per 100,000 doses $(33,34)$. This disorder is caused by autoantibodies against platelets and/or megakaryocytes. Although, in children, ITP in general mostly has a benign, self-limited course (acute ITP), some children do progress to chronic ITP requiring long-term immunosuppression treatment and/or splenectomy. Vaccine-triggered ITP is most frequently (>90\%) found to be acute, benign and self-limited (35). Specific predisposing factors are, as yet, unknown. Risk of ITP has not prompted experts to alter vaccination recommendations, mainly because infections, including measles, entail a significantly higher risk of triggering the same disorder in susceptible children (36). The overall effect of MMR vaccine on the risk of childhood ITP is, therefore, protective.

\section{Myopericarditis and Smallpox Vaccination}

Finally, even though smallpox vaccination is no longer performed (except in certain military settings) because the disease has been eradicated, it is worth mentioning myopericarditis that followed the administration of smallpox vaccine with an estimated incidence of about 8 per 100,000 administered doses (37). This rate was sufficient to conclude that a causal relationship is probably present, since the odds ratio compared to sex- and age-matched non-vaccinated persons was 3.6 (95\% CI, 3.3-4.1). In addition to providing important vaccine safety information, this finding is a useful illustration of how a properly applied scientific methodology can reliably detect an epidemiological signal supporting a causal relationship between a vaccine and an autoimmune phenomenon, casting additional light to all the countless instances when, after an avalanche of reports on some potential vaccine-autoimmunity connection, the same rigorous methodology found the corresponding epidemiological signal to be weak or absent, effectively disproving a causal relationship.

\section{Vaccination-Induced Autoimmunity: Proposed Causal Connection}

The space allowed for this review precludes any attempt at enumeration of all the reported cases of temporal association between vaccine administration and autoimmunity. Examples below are intended purely as a representative illustration.

\section{Narcolepsy}

In 2009, at the height of the "Mexican" H1N1 influenza pandemic, some countries in Europe reported a sudden increase in the incidence of narcolepsy (38). This disorder is associated with a strong genetic predisposition, most commonly HLA DQB1*06:02, although other gene polymorphisms may also play a role (39). Antibodies to hypokretin, a protein known to participate in maintaining wakefulness, have been proposed as a potential causative mechanism. Epidemiological evidence of association between narcolepsy and influenza vaccination was initially deemed sufficiently strong to grant compensation to affected persons. However, follow-up investigations yielded conflicting results (40). At the time of writing, many important questions still await an answer and the issue of vaccine-induced narcolepsy remains largely unresolved $(41,42)$.

\section{Multiple Sclerosis}

Few examples illustrate so vividly the discrepancy between the general impression imparted by a multitude of published case reports, 
case series and small initial studies and the results of comprehensive meta-analyses of large studies as does the example of putative connection between hepatitis $\mathrm{B}$ vaccination and multiple sclerosis (MS). In addition to several case reports and series, a frequently cited French case-control study of 121 subjects found an odds ratio of 1.4 (95\% CI, 0.5-4.3) for exposure to hepatitis B vaccine within 60 days prior to onset of first demyelination episode in MS (43). However, many large and rigorous studies subsequently failed to confirm such causal connection $(44,45,46)$. Comprehensive analysis of data available at this time does not support the hypothesis of a causal role for any vaccine in the etiopathogenesis of MS, nor a substantial risk of exacerbation of existing disease by vaccination (47).

\section{Diabetes mellitus type 1}

Based on numerous case reports and series, a potential causal connection of type 1 diabetes (T1D) and vaccination has frequently been invoked. However, as was the case in MS, controlled studies did not confirm such connection, and a slight protective effect, probably due to elimination of infections that could promote T1D development, has been found instead (48).

\section{Systemic (Autoimmune) Vasculitis}

In one patient with antineutrophil cytoplasmic antibodies (ANCA)-associated vasculitis arising after influenza vaccination, a careful study of the patient's peripheral blood mononuclear cells in vitro demonstrated the production of pathogenic anti-proteinase 3 autoantibodies upon stimulation with influenza vaccines containing viral RNA (49). This finding supports the hypothesis that influenza vaccine, similarly to influenza infection, may trigger autoantibody production and consequent vasculitis by acting on innate immunity via Toll-like receptor 7 that binds viral RNA. However, the connection appears to be confined to individual case(s) and cannot be a frequent occurrence even in predisposed subjects, since large studies failed to find a statistical association between either initiation or exacerbation of ANCA-associated vasculitis and influenza vaccination (50, 51,52 ). Systemic vasculitides associated with other vaccines also appear to be quite rare (53). The same has been concluded for Kawasaki disease (54).

\section{Human Papilloma Virus Vaccination and Autoimmunity}

There are many reports in the literature substantiating instances of initiation or exacerbation of various autoimmune diseases after human papilloma virus (HPV) vaccination, including cases of acute disseminated encephalomyelitis (55), autoimmune pancreatitis (56), immune thrombocytopenia (57) and systemic lupus erythematosus (58). The list is by no means exhaustive, and a remarkably bitter international debate continues, sometimes including court litigation for alleged health damage inflicted by HPV vaccine. A frequently cited fact in this debate is that as many as 21,303 reports of adverse events after immunization by HPV vaccines have been submitted to the US Vaccine Adverse Events Reporting System (VAERS) since HPV vaccines were introduced (2006 and 2009 for quadrivalent and bivalent vaccines, respectively), including 3,310 reports of serious adverse events, 69 reports of death, and 760 reports of permanent disability. However, since VAERS is an open system of passive surveillance, intended solely for early detection of potential epidemiological patterns, it is impossible to deduce directly from these data how many of the reports reflect autoimmune phenomena, or indeed how many of the reported events were actually causally connected to HPV vaccination. One must al- 
ways keep in mind that a certain number of cases of temporal connection between HPV vaccine and autoimmune disease onset or exacerbation is almost inevitable to occur, given that this vaccine is offered to a population with relatively high incidence of AID, and at an age not far removed from the age of peak incidence of some AID, such as MS. Reports of such instances, therefore, need to be interpreted in the proper context. Importantly, vaccine vigilance data and controlled studies where risk of AID was compared between HPV-vaccinated and matched unvaccinated populations failed to find any significant difference in risk of developing an AID, suggesting that causal connection between HPV vaccine and autoimmunity must, at the very most, be a rare occurrence (59) and that HPV vaccines in use continue to display a favorable safety profile $(60,61)$. Addressing the ongoing public controversy, the Global Advisory Committee of the World Health Organization, after carefully considering all available evidence, concluded that there is no proof of significant risk of autoimmune adverse reactions to HPV immunization, with the possible exception of GuillainBarré syndrome (62).

\section{"Autoimmune/inflammatory Syndrome Induced by Adjuvants"}

The term "Autoimmune/inflammatory Syndrome Induced by Adjuvants" (ASIA) has recently been proposed to denote a clinical entity encompassing a wide variety of presumed autoimmune or inflammatory reactions to immunization (63). The proposed diagnostic criteria for ASIA are quite inclusive, assuring high sensitivity, but raising the key question of specificity, i.e., the risk of falsely concluding that occurrence of an autoimmune phemonenon in the appropriate temporal window after administration of a vaccine represents a bona fide causal connection. While potentially useful in the generation of research hypotheses, these proposed criteria are, therefore, no substitute for rigorous studies designed to test such hypotheses and fulfilment of such criteria should by no means be considered "proof" that a particular phenomenon, autoimmune or otherwise, was indeed caused by a vaccine, as recently highlighted by a systematic review of 27 animal, epidemiological and case studies related to ASIA (64).

\section{Vaccination in Patients with Autoimmune Disorders}

Since infectious diseases, including vaccinepreventable diseases, are among the most common triggers, and possibly inducers, of autoimmune disorders, it is plausible to expect a net protective effect of vaccination (65). On the other hand, there is currently a widespread concern that in predisposed persons vaccination may entail a risk of triggering an autoimmune disorder, as well as exacerbating the course of an existing disorder (66). It is therefore of note that, individual case reports and case series notwithstanding, a vast body of statistic evidence appears to suggest that initiation or exacerbation of AID by vaccines is a rare occurrence, even in persons carrying one or more potential risk factors. Additional issues to be explored include potential effects of immunosuppressive therapy on the risk of developing an autoimmune reaction to a vaccine (i.e., whether immunosuppression could be somewhat protective in this regard) and whether the overall risk of new autoimmune phenomena in patients with AID may be modified by repeated antigenic challenge.

Systemic lupus erythematosus (SLE), a prototypical systemic autoimmune disorder, may serve as an example. Some animal model data appear to support a potential detrimental effect of vaccination on SLE patients, such as the finding of exacerbation induced by $H B V$ vaccine in a murine SLE model (please see reference 8 above). However, this 
is not reflected by clinical studies of vaccine safety in SLE. One systematic review (67) found vaccination to be safe in SLE, with the well-known exception of administering live vaccines in the setting of immunosuppression (which is generally contraindicated), although immunization may achieve a reduced level of protection in deeply immunosuppressed persons, such as people undergoing immunosuppressive treatment for AID, particularly by potent anti-cytokine agents. Another recent review confirmed infections as important triggers of exacerbations in SLE and found vaccination to be safe and effective in SLE patients (68). Specifically, vaccination against pneumococcus and influenza has been shown not to increase the risk of SLE flares, while prevention of these disorders may, in turn, prevent infection-associated disease flares $(69,70)$. A recent review of issues bearing on vaccination of SLE patients, based on experience in Portugal, concluded that any risk of triggering SLE exacerbation by vaccines is low, and largely offset by benefits of protection from certain infections, fully supporting the current recommendations (71). This review also endorsed the precaution taken by most clinicians not to vaccinate patients in periods of elevated disease activity, but warned against the widespread practice of baselessly withholding vaccination in people suffering from AID. Conclusions reached for SLE may be expected to be a fortiori applicable to other AID as well. Indeed, studies looking into most other AID yielded similar results, with the sum of evidence reflected in current worldwide recommendations for vaccination of patients with AID (72).

\section{Towards the Future: Can Vaccine- Triggered Autoimmunity Be Predicted?}

From a clinical standpoint, the most important common denominator of most, if not all, immunological adverse reactions to vaccines is the presence of individual predisposition toward a specific reaction to a specific trigger (66). Although it is currently, in most cases, impossible to screen for such predisposing factors, rapid development of technology could, in the near future, allow doctors to know in advance who will be at risk of an immunologically mediated idiosyncratic adverse event. A wide array of powerful technologies (reviewed in 73) is bringing humanity ever closer to such capabilities, and results of deep investigation of individual immune responses to both infections and vaccines are increasingly being published. Given the importance of individual variations in characteristics of the immune system for successful prevention and treatment of many medical conditions, including adverse reactions to drugs or vaccines, the emerging field of systems immunology is virtually guaranteed to be an essential part of comprehensive, patient-centered, science-based medicine of the future.

\section{Conclusion}

Autoimmunity triggered by vaccination is a documented, but rare occurrence. The risk of vaccine-related autoimmunity is by orders of magnitude lower than that of autoimmunity triggered by infectious disease. Since the latter includes vaccine-preventable infections, for most autoimmune conditions there is a net protective effect of vaccination. Current evidence supports administering appropriate vaccines to people suffering from autoimmune disorders in the absence of specific contraindications, such as deep immunosuppression for live vaccines. More research into potential mechanisms of vaccine-initiated autoimmune processes is warranted, and future scientific technology and methodology may enable immunological screening of subjects prior to immunization in order to avoid 
administering particular vaccines to individuals who are strongly predisposed toward a detrimental reaction.

Acknowledgement: The author received support from the Ministry of Education, Science and Technological Development of the Republic of Serbia, Grant No. 41004. The author wishes to thank Prof. Dr. Zoran Radovanović and Dr. Slavko Mojsilović for their invaluable comments and highly fruitful discussion.

Conflict of interest: The author declares that he has no conflict of interest.

\section{References}

1. Shoenfeld Y, Agmon-Levin N, Tomljenovic L (eds). Vaccines and autoimmunity. Wiley-Blackwell, 2015.

2. Salemi S, D’Amelio R. Could autoimmunity be induced by vaccination? Int Rev Immunol. 2010;29(3):247-69.

3. Salemi S, D'Amelio R. Are anti-infectious vaccinations safe and effective in patients with autoimmunity? Int Rev Immunol. 2010;29(3):270-314.

4. Jensen KJ, Benn CS, van Crevel R. Unravelling the nature of non-specific effects of vaccines - A challenge for innate immunologists. Semin Immunol. 2016;28(4):377-83.

5. Ruiz JT, Luján L, Blank M, Shoenfeld Y. Adjuvants- and vaccines-induced autoimmunity: animal models. Immunol Res. 2016 [Epub ahead of print]. DOI:10.1007/s12026-016-8819-5.

6. Kuroda Y, Nacionales DC, Akaogi J, Reeves WH, Satoh M. Autoimmunity induced by adjuvant hydrocarbon oil components of vaccine. Biomed Pharmacother. 2004; 58(5):325-37.

7. Aratani S, Fujita H, Kuroiwa Y, Usui C, Yokota S, Nakamura I, Nishioka K, Nakajima T. Murine hypothalamic destruction with vascular cell apoptosis subsequent to combined administration of human papilloma virus vaccine and pertussis toxin. Sci Rep. 2016; 6:36943.

8. Agmon-Levin N, Arango MT, Kivity S, Katzav A, Gilburd B, Blank M, Tomer N, Volkov A, Barshack I, Chapman J, Shoenfeld Y. Immunization with hepatitis B vaccine accelerates SLE-like disease in a murine model. J Autoimmun. 2014; 54:21-32.

9. Shaw CA, Tomljenovic L. Aluminum in the central nervous system (CNS): toxicity in humans and animals, vaccine adjuvants, and autoimmunity. Immunol Res. 2013; 56(2-3):304-16.

10. Gherardi RK, Eidi H, Crépeaux G, Authier FJ, Cadusseau J. Biopersistence and brain translocation of aluminum adjuvants of vaccines. Front Neuro.l 2015;6:4.

11. Inbar R, Weiss R, Tomljenovic L, Arango MT, Deri $\mathrm{Y}$, Shaw CA, et al. Behavioral abnormalities in female mice following administration of aluminum adjuvants and the human papillomavirus (HPV) vaccine Gardasil. Immunol Res. 2016 [Epub ahead of print]; DOI: 10.1007/s12026-016-8826-6.

12. World Health Organization [homepage on the Internet]. Statement from the Global Advisory Committee on Vaccine Safety on aluminium-containing vaccines. October 2008 [last updated: October 2008; last reviewed: December 2008; cited: February 2017]. Available at: http://www.who.int/ vaccine_safety/committee/topics/aluminium/statement_112002/en/.

13. Gołoś A, Lutyńska A. Aluminium-adjuvanted vaccines - a review of the current state of knowledge. Przegl Epidemiol. 2015; 69(4):731-4, 871-4.

14. Stassijns J, Bollaerts K, Baay M, Verstraeten T. A systematic review and meta-analysis on the safety of newly adjuvanted vaccines among children. Vaccine 2016; 34:714-22.

15. Melnick SC. Role of infection in the GuillainBarre syndrome. Neurol Neurosurg Psychiatry. 1964;27:395-407.

16. Fujinami RS, Oldstone MB. Amino acid homology between the encephalitogenic site of myelin basic protein and virus: mechanism for autoimmunity. Science. 1985;230:1043-45.

17. Kanduc D, Shoenfeld Y. From HBV to HPV: Designing vaccines for extensive and intensive vaccination campaigns worldwide. Autoimmun Rev. 2016;15(1):1054-61.

18. Chen RT, Pless R, Destefano F. Epidemiology of autoimmune reactions induced by vaccination. J Autoimmun. 2001;16:309-18.

19. Gross DM, Forsthuber T, Tary-Lehmann M, Etling C, Ito K, Nagy ZA, et al. Identification of LFA-1 as a candidate autoantigen in treatment-resistant Lyme arthritis. Science. 1998;281:703-6.

20. Stojanović M, Petrušić V, Zivković I, Inić-Kanada A, Stojićević I, Marinković E, et al. Role of molecular mimicry and polyclonal cell activation in the induction of pathogenic $\beta 2$-glycoprotein I-directed immune response in Balb/c mice upon hyperim- 
munization with tetanus toxoid. Immunol Res. 2013; 56(1):20-31.

21. Bogdanos DP, Smith H, Ma Y, Baum H, MieliVergani G, Vergani D. A study of molecular mimicry and immunological cross-reactivity between hepatitis B surface antigen and myelin mimics. Clin Dev Immunol. 2005; 12(3):217-24.

22. Kanduc D. Potential cross-reactivity between HPV16 L1 protein and sudden death-associated antigens. J Exp Ther Oncol. 2011;9(2):159-65.

23. Schattner A. Consequence or coincidence? The occurrence, pathogenesis and significance of autoimmune manifestations after viral vaccines. Vaccine 2005;23:3876-86.

24. Toplak N, Kveder T, Trampus-Bakija A, Subelj V, Cucnik S, Avcin T. Autoimmune response following annual influenza vaccination in 92 apparently healthy adults. Autoimmune Rev. 2008;8(2):134-8.

25. Stuart G, Krikorian KS. The neuro-paralytic accidents of anti-rabies treatment. Ann Trop Med Parasitol. 1928;22:327-77.

26. Wakerley BR, Yuki N. Infectious and noninfectious triggers in Guillain-Barré syndrome. Expert Rev Clin Immunol. 2013;9(7):627-39.

27. Winer JB. An update in Guillain-Barré syndrome. Autoimmune Dis 2014;2014:793024.

28. Schoenfeld Y, Aron-Maor A. Vaccination and autoimmunity - 'vaccinosis': a dangerous liaison? J Autoimmun. 2000;14(1):1-10.

29. Schonberger LB, Bregman DJ, Sullivan-Bolyai JZ, Keenlyside RA, Ziegler DW, Retailliau HF, et al. Guillain-Barre syndrome following vaccination in the National Influenza Immunization Program, United States, 1976-1977. Am J Epidemiol. 1979;110(2):105-23.

30. Iqbal S, Li R, Gargiullo P, Vellozzi C. Relationship between Guillain-Barré syndrome, influenza-related hospitalizations, and influenza vaccine coverage. Vaccine. 2015;33(17):2045-9.

31. Karussis D, Petrou P. The spectrum of post-vaccination inflammatory CNS demyelinating syndromes. Autoimmun Rev. 2014;13(3):215-24.

32. Stübgen JP. Immune-mediated myelitis following hepatitis B vaccination. Autoimmune Rev. 2012;12(2):144-9.

33. O’Leary ST, Glanz JM, McClure DL, Akhtar A, Daley MF, Nakasato C, et al. The risk of immune thrombocytopenic purpura in children and adolescents. Pediatrics. 2012;129(2):248-55.
34. Cecinati V, Principi N, Brescia L, Giordano P, Esposito $S$. Vaccine administration and the development of immune thrombocytopenic purpura in children. Hum Vaccin Immunother. 2013;9(5):1158-62.

35. Mantadakis E, Farmaki E, Buchanan GR. Thrombocytopenic purpura after measles-mumps-rubella vaccination: a systematic review of the literature and guidance for management. J Pediatr. 2010;156(4):623-8.

36. Bester JC. Measles and Measles Vaccination: A Review. JAMA Pediatr. 2016;170(12):1209-15.

37. Halsell JS, Riddle JR, Atwood JE, Gardner P, Shope R, Poland GA, et al. Department of Defense Smallpox Vaccination Clinical Evaluation Team. Myopericarditis following smallpox vaccination among vaccinia-naïve US millitary personnel. JAMA. 2003;298:3283-9.

38. Wijnans L, Lecomte C, de Vries C, Weibel D, Sammon C, Hviid A, et al. The incidence of narkolepsy in Europe: before, during, and after the influenza $\mathrm{A}(\mathrm{H} 1 \mathrm{~N} 1) \mathrm{pdm} 09$ pandemic and vaccination campaigns. Vaccine. 2013;31(8):1246-54.

39. Singh AK, Mahlios J, Mignot E. Genetic association, seasonal infections and autoimmune basis of narcolepsy. J Autoimmun. 2013;43:26-31.

40. Melén K, Partinen M, Tynell J, Silanpää M, Himanen SL, Saarenpää-Heikkilä O, et al. No serological evidence of influenza A H1N1pdm09 virus infection as a contributing factor in childhood narcolepsy after Pandemrix vaccination capmaign in Finland. PLoS One. 2013;8(8):e68402.

41. Sturkenboom MC. The narcolepsy-pandemic influenza story: can the truth ever be unraveled? Vaccine. 2015;33(Suppl 2):B6-B13.

42. Verstraeten T, Cohet C, Dos Santos G, Ferreira GL, Bollaerts K, Bauchau V, et al. Pandemrix and narcolepsy: A critical appraisal of the observational studies. Hum Vaccin Immunother. 2016;12(1):187-93.

43. Touzé E, Gout O, Verdier-Teillefer MD, Lyon-Caen $\mathrm{O}$, Alpérovitch A. The first epizode of central nervous system demyelization and hepatitis $B$ virus vaccination. Rev Neurol (Paris). 2000;156(3):242-6.

44. Ascherio A, Zhang SM, Hernán MA, Olek MJ, Coplan PM, Brodovicz K, et al. Vaccination and the risk of multiple sclerosis. N Engl J Med. 2001; 344(5):327-32.

45. Martínez-Sernández V, Figueiras A. Central nervous system demyelinating diseases and recombinant hepatitis B vaccination: a critical system- 
atic review of scientific production. J Neurol. 2013;260(8):1951-9.

46. Mailand MT, Frederiksen JL. Vaccines and multiple sclerosis: a systematic review. J Neurol 2016; [Epub ahead of print]; PMID:27604618; DOI:10.1007/ s00415-016-8263-4.

47. Williamson EML, Chahin S, Berger JR. Vaccines in multiple sclerosis. Curr Neurol Neurosci Rep. 2016;16:36.

48. Morgan E, Halliday SR, Campbell GR, Cardwell CR, Patterson CC. Vaccination and childhood type 1 diabetes mellitus: a meta-analysis of observational studies. Diabetologia. 2016;59:237-43.

49. Jeffs LS, Nitschke J, Terwaert JW, Peh CA, Hurtado PR. Viral RNA in the influenza vaccine may have contributed to the development of ANCAassociated vasculitis in a patient following immunisation. Clin Rheumatol. 2016;35(4):943-51.

50. Stassen PM, Sanders JS, Kallenberg CG, Stegeman CA. Influenza vaccination does not result in an increase in relapses in patients with ANCAassociated vasculitis. Nephrol Dial Transplant. 2008;23(2):654-8.

51. Mahajan D, Roomiani I, Gold MS, Lawrence GL, McIntyre PB, Menzies RI. Annual report, surveillance of adverse events following immunisation in Australia. Commun Dis Intell. 2009;34(3):259-6.

52. Vellozi C, Burwen DR, Dobardzic A, Ball R, Walton K, Haber P. Safety of trivalent inactivated influenza vaccines in adults: background for pandemic influenza vaccine safety monitoring. Vaccine. 27(15);2114-20.

53. Bonetto C, Trotta F, Felicetti P, Alarcón GS, Santuccio C, Bachtiar NS, et al. Vasculitis as an adverse event following immunization - Systematic literature review. Vaccine. 2016;34(51):6641-51.

54. Abrams JY, Weintraub ES, Baggs JM, McCarthy NL, Schonberger LB, Lee GM, et al. Childhood vaccines and Kawasaki disease, Vaccine Safety Datalink, 1996-2006. Vaccine. 2015;33:382-7.

55. Sekiguchi K, Yasui, Kowa H, Kanda F, Toda T. Two cases of acute disseminated encephalomyelitis following vaccination against human papilloma virus. Intern Med. 2016;55(21):3181-4.

56. Bizjak M, Bruck O, Prapotnik S, Dahan S, Shoenfeld Y. Pancreatitis after human papilloma vaccination: a matter of molecular mimicry. Immunol Res. 2016; Epub ahead of print; doi:10.1007/s12026016-8823-9.
57. Bizjak M, Bruck O, Kanduc D, Prapotnik S, Shoenfeld Y. Vaccinations and secondary immune thrombocytopenia with antiphospholipid antibodies by human papillomavirus vaccine. Semin Hematol. 2016;53 (Suppl 1):S48-50.

58. Ito H, Noda K, Hirai K, Ukichi T, Furuya K, Kurosaka D. A case of systemic lupus erythematosus (SLE) following human papillomavirus (HPV) vaccination. Nihon Rinsho Meneki Gakkai Kaishi. 2016; 39(2):145-9.

59. Pellegrino P, Carnovale C, Pozzi M, Antoniazzi S, Perrone V, Salviati D, et al. On the relationship between human papilloma virus vaccine and autoimmune diseases. Autoimmune Rev. 2014;13(7):73641.

60. Vichnin M, Bonnani P, Klein NP, Garland SM, Block SL, Kjaer SK, et al. An overview of quadrivalent human papillomavirus vaccine safety: 2006 to 2015. Pediatr Infect Dis J. 2015;34(9):983-91.

61. De Vincenzo R, Conte C, Ricci C, Scambia G, Capelli G. Long-term efficacy and safety of human papillomavirus vaccination. Int J Womens Health. 2014;6:999-1010.

62. World Health Organization [homepage on the Internet]. Safety of HPV vaccines. Extract from report of GACVS meeting of 2-3 december 2015, published in the WHO Weekly Epidemiological Record of 22 January 2016 [last updated/reviewed: January 2016; cited: February 2017]. Available at: http://www.who.int/vaccine_safety/committee/ topics/hpv/Dec_2015/en/

63. Shoenfeld Y, Agmon-Levin N. 'ASIA' - Autoimmune/inflammatory syndrome induced by adjuvants. J Autoimmun. 2011;36(1):4-8.

64. Hawkes D, Benhamu J, Sidwell T, Miles R, Dunlop RA. Revisiting adverse reactions to vaccines: a critical appraisal of Autoimmune Syndrome Induced by Adjuvants (ASIA). J Autoimmun. 2015;59:7784.

65. McKinnon JE, Maksimowicz-McKinnon K. Autoimmune disease and vaccination: impact on infectious disease prevention and a look at future applications. Transl Res. 2016;167:46-60.

66. Soriano A, Nesher G, Shoenfeld Y. Predicting postvaccination autoimmunity: Who might be at risk? Pharmacol Res. 2015;92:18-22.

67. Millet A, Decaux O, Perlat A, Grosbois B, Jego P. Systemic lupus erythematosus and vaccination. Eur J Intern Med. 2009;20(3):236-41. 
68. Pasoto SG, Ribeiro AC, Bonfa E. Update on infections and vaccinations in systemic lupus erythematosus and Sjögren's syndrome. Curr Opin Rheumatol. 2014;26(5):528-37.

69. Abu-Shakra M, Zalmanson S, Neumann L, Flusser D, Sukenik S, Buskila D. Influenza virus vaccination of patients with systemic lupus erythematosus: effects on disease activity. J Rheumatol. 2000;27:1681-5.

70. Elkayam O, Paran D, Burke M, Zakut V, Ben-Yitshak R, Litinsky I, et al. Pneumococcal vaccination of patients with systemic lupus erythematosus: effects on generation of autoantibodies. Autoimmunity. 2005;38:493-6.
71. Moraes-Fontes MF, Antunes AM, Gruner H, Riso N. Vaccination of adult patients with systemic lupus erythematosus in Portugal. Int J Rheumatol. $2016 ; 2845617$.

72. Bühler S, Eperon G, Ribi C, Kyburz D, van Gompel F, Visser LG, et al. Vaccination recommendations for adult patients with autoimmune inflammatory rheumatic diseases. Swiss Med Wkly. 2015;145:w14159.

73. Furman D, Davis MM. New approaches to understanding the immune response to vaccination and infection. Vaccine. 2015;33(40):5271-81. 